\title{
ESTUDIO CUALITATIVO DE LAS DIFERENCIAS DE GÉNERO EN LA ELECCIÓN DE OPCIONES ACADÉMICAS EN LOS ESTUDIANTES DEL BACHILLERATO CIENTÍFICO-TÉCNICO
}

Gender differences in academic decisions of high school students who choose scientific-technological subjets: a qualitative study

Étude qualitative des différences de genre dans le choix des options académiques par les élèves $d u$ baccalauréat scientifique et technologuique

María del Carmen Rodríguez Méndez, José Vicente PeÑa Calvo y Omar GARCÍA PÉREZ

Universidad de Oviedo. Facultad de Formación del Profesorado y Educación. Departamento de Ciencias de la Educación. C/ Aniceto Sela, s/n. 33005 Oviedo.carmenrm@uniovi.es; vipe@uniovi.es; garciaomar@uniovi.es

Fecha de recepción: enero de 2016

Fecha de aceptación: marzo de 2016

RESUMEN

El artículo analiza las causas que ayudan a explicar las diferencias de género en las elecciones académicas a partir de la opinión de los progenitores, docentes y estudiantes. Se ha usado una metodología cualitativa basada en el uso de grupos de discusión. Se organizaron cinco grupos con estudiantes que cursan la modalidad de bachillerato científico-técnico en distintas instituciones de educación 
secundaria del Principado de Asturias, también se realizaron seis grupos con docentes y dos grupos con progenitores. Los resultados permiten concluir que sigue existiendo un sesgo de género en la elección. La razón fundamental que indican los estudiantes para hacer sus elecciones tiene que ver con lo que hemos denominado la «ideología del gusto innato", pues eligen los estudios porque les gustan o sienten vocación hacia ellos, reforzando los estereotipos de género. El artículo finaliza con unas orientaciones para desarrollar una orientación vocacional no sesgada por razón de género.

Palabras clave: educación secundaria; orientación educativa; género; elección de estudios; análisis de contenido.

\section{SUMMARY}

The current paper analyses parents', teachers' and students' opinions about the academic decisions. A qualitative methodology based on focus groups has been used. There have been developed five focus groups with students who are studying Scientific/Technological Senior Secondary Education in Asturias (Spain), six focus groups with teachers and two with parents. Results show that there still exist gender bias in the elections. Moreover, the most important reason to explain students' options is what we have called "the ideology of innate liking" that is, preferring the studies because they like them or because they feel a vocation towards them. The paper ends with some recommendations to develop a vocational counselling free form gender stereotypes.

Key words: secondary education; educational guidance; gender; choice of studies; content analysis.

\section{SOMMAIRE}

L'article analyse les causes qui contribuent à expliquer les différences entre les sexes dans les choix des études universitaires, selon les points de vue des parents, des enseignants et des apprenants. Il a été utilisé une méthodologie qualitative basée sur l'emploi de groupes de discussion. On a organisé cinq groupes avec des apprenants du baccalauréat de la modalité scientifique et technique de différents établissements de l'éducation secondaire des Asturies, ainsi que six groupes avec des enseignants, et deux autres avec des parents. Les résultats suggèrent qu'il y a encore un parti pris sexiste dans le choix. La justification donnée par les apprenants, à propos de leurs choix, est en rapport avec ce que nous avons appelé " l'idéologie du goût inné ", car ils choisissent les études en fonction de leurs goûts, ou par vocation, tout en renforçant les stéréotypes de genre. L'article conclut avec des lignes directrices pour développer une orientation professionnelle libre des préjugés sexistes.

Mots clés: l'enseignement secondaire; l'orientation de l'éducation; le genre; le choix des études; l'analyse de contenu. 
M. C. RODRÍGUEZ MÉNDEZ, J. V. PEÑA CALVO Y O. GARCÍA PÉREZ

\section{INTRODUCCIÓN}

Si analizamos las estadísticas de elección de opciones académicas en el sistema universitario español observamos que siguen persistiendo las diferencias de género. Así, para el curso académico 2013/2014 el porcentaje de mujeres matriculadas en la Universidad española ascendió al 54.2\% del total (MECD, 2016). No obstante, también se observó la persistencia de claros sesgos de género en la elección de las carreras. Así, para el mismo curso académico, las mujeres fueron amplia mayoría en la rama de Ciencias de la Salud (69.7\% del total), de Arte y Humanidades (61.3\% del total) y de Ciencias Sociales y Jurídicas (60.7\% del total); no obstante, su presencia fue mucho más exigua en la rama de Ingeniería y Arquitectura (25.8\% del total). Asimismo, se observó un equilibrio de sexo en lo que respecta a la rama de conocimiento de Ciencias, pues el $51.7 \%$ del alumnado es femenino y el $48.3 \%$ es masculino. Estos resultados estadísticos también se encuentran en otros países europeos. Investigaciones realizadas en Gran Bretaña (Rodd y Bartholomew, 2006), Australia (Anderson, Lankshear, Timms y Courtney, 2008), países escandinavos (Brandell, Leder y Nyström, 2007; Brandell y Staberg, 2008) o EE. UU. (Frome, Alfeld, Eccles y Barber, 2006; Zarret y Malanchuk, 2005) concluyen que las mujeres están infrarrepresentadas en los estudios tecnológicos y de ingeniería.

En este contexto, el estudio que presentamos analiza la persistencia de las diferencias de género en la elección académica de estudiantes que cursan primero de bachillerato científico-tecnológico. Se ha usado una metodología cualitativa, basada en el uso de grupos de discusión, para conocer la opinión de progenitores, estudiantes y docentes, sobre las causas de la elección diferencial por razón de género.

\section{ANTECEDENTES Y FUNDAMENTACIÓN}

El análisis del proceso de elección diferencial de elecciones académicas es muy complejo porque influyen multitud de factores personales y sociales. Con frecuencia, chicas y chicos recurren a justificaciones basadas en el gusto o la vocación que sienten por determinadas opciones vocacionales (Cortés y Conchado, 2012; Santana y Feliciano, 2009; Santana, Feliciano y Jiménez, 2012). Así, cuando se les pregunta cuáles son sus materias preferidas se observa la persistencia de los estereotipos de género, ellas prefieren cursar asignaturas de biología, más que de física o matemáticas, aludiendo a motivos relacionados con la ayuda a personas o animales. Parece que hay un mayor interés de las alumnas hacia una ciencia contextualizada en la vida cotidiana y las necesidades de las personas, y un mayor interés de los varones hacia aparatos y máquinas (Reid y Skryabina, 2003; Stewart, 1998). En este mismo sentido, Sáinz y López-Sáez (2010) constatan que las chicas tienen actitudes más positivas hacia las habilidades sociales que necesita un profesional de la tecnología/informática. 
Igualmente, las investigaciones realizadas en nuestro país por Vázquez y Manassero (2007, 2008a, 2008b, 2009) constatan que los chicos dan más importancia a los rasgos manuales o tecnológicos de los trabajos del campo científico-tecnológico. Asimismo, las chicas puntúan significativamente más bajo que los chicos en la opción: tener un trabajo tecnológico. Del mismo modo, cuando se les pregunta si les gustaría conseguir un trabajo en tecnología, ellos lo valoran positivamente y ellas de forma negativa; del mismo modo, las chicas rechazan más la vocación de llegar a ser científicas.

También debemos comentar que los estudios no son concluyentes respecto a la existencia de estereotipos de género que perpetúan el pensamiento tácito de que determinadas materias no son apropiadas para las mujeres. No obstante, sí hay mayor unanimidad en la conclusión de que los chicos tienen una visión más acorde al estereotipo y que ellas están más dispuestas a considerar que las matemáticas o la informática no son "masculinas» y que también pueden ser científicas o tecnólogas (Bovée, Voogt y Meelinssen, 2007; Brandell y Staberg, 2008; Johnson, Stone, Phillips, 2008; Meelissen y Drent, 2008; Papastergiou, 2008). Si tomamos en cuenta esta conclusión, se demuestra la paradoja "Yo no puedo, pero nosotras sí podemos». Esta paradoja indica que las chicas niegan el estereotipo, pero les cuesta identificarse personalmente con estas materias (véanse los estudios analizados por Singh, Allen, Scheckler y Darlington, 2007).

Así, respecto al interés por las matemáticas o la tecnología, los estudios han comprobado que los varones manifiestan más interés en estas materias (Anderson, 2005; Anderson et al., 2008; Linver y Davis-Kean, 2005; Rodríguez y Angulo, 2006; Papastergiou, 2008). En esta línea, también destacamos el estudio de López-Sáez, Puertas y Sáinz (2011) que determina que los estudiantes de secundaria de su muestra perciben que las modalidades de Bachillerato más femeninas fueron las Humanidades y las Ciencias Sociales, seguidas de las Ciencias Naturales y de la Salud. Asimismo, el bachillerato tecnológico es visto como altamente masculino.

Sin lugar a dudas, a estos factores personales hay que añadir la influencia ejercida por el contexto social que rodea a los adolescentes. Ellos y ellas observan un mundo marcado por las diferencias de género en las profesiones y este modelado social actúa como un factor que influye en la elección. En este sentido, Sáinz, Meneses y López (2014) concluyen que los estudiantes de secundaria que participaron en su estudio tenían una imagen positiva de los profesionales que se dedicaban al mundo de la tecnología, pero señalaron pocos modelos de rol femeninos en este ámbito profesional. Para los varones están disponibles muchos más modelos masculinos que han tenido éxito en el campo tecnológico. Tanto en el contexto escolar (Clark, 2005; Erwin y Maurutto, 1998; Fox y Soller, 2001; Meelissen et al., 2008; Reinen y Plomp, 1997) como en el ámbito familiar tienen a su disposición varones que trabajan en esos campos profesionales (Erwin et al., 1998; López, 1995; Zeldin y Pajares, 2000; Zeldin, Britner y Pajares, 2008).

A ello debemos añadir que puesto que la sociedad ha definido los patrones de conducta socialmente deseables para cada uno de los sexos, varones y mujeres 
reciben mensajes positivos y negativos cuando realizan determinadas elecciones académicas. En este sentido, los estudios cualitativos indican que muchas mujeres que han realizado carreras universitarias en el dominio científico-tecnológico reconocen la relevancia del apoyo de personas allegadas para elegir y perseverar en su carrera académica (Clegg y Trayhurn, 1999; Erwin et al., 1998; Scott y Mallinckrodt, 2005; Zeldin et al., 2000). Por otra parte, en el estudio de Sainz, Palmen y García-Cuesta (2012) se trabajó con padres/madres en grupos de discusión y estos proporcionaron diversas explicaciones a la menor presencia femenina en la modalidad tecnológica: menor interés y aptitudes de las chicas en estos estudios, existencia de una segregación ocupacional en las profesiones vinculadas a esta modalidad, asunción de que hay ocupaciones más apropiadas para las chicas y otras para los chicos, así como la ausencia de modelos femeninos en determinadas ocupaciones. Igualmente, todos los padres/madres reconocieron que el sistema educativo, los medios de comunicación y la familia son influencias socializadoras que median en los procesos de decisión vocacional. De todos modos, la inmensa mayoría de los progenitores también indicaron que su influencia era poco importante y que sus hijos/as eran libres de elegir su carrera académica de acuerdo a sus intereses.

En lo que respecta a los docentes, también se obtienen resultados interesantes que demuestran que tienen distintas expectativas respecto al éxito académico de chicos y chicas en distintas materias. Así, los niños tienden a ser elegidos como los estudiantes más competentes en matemáticas. Atribuyen el alto rendimiento de las chicas a su habilidad y esfuerzo, mientras que el de ellos se atribuye a su capacidad o talento innato. Igualmente, los docentes consideran que los chicos que son buenos en matemáticas son lógicos, persistentes y competitivos y que las chicas con éxito también lo son, pero en menor grado. Asimismo se considera que ellos exhiben un aprendizaje más autónomo, son más independientes y disfrutan más con las matemáticas (Sáinz et al., 2012. Véase también la revisión bibliográfica de Li, 1999).

En este contexto, la investigación que presentamos ha sido realizada en el Principado de Asturias para conocer las percepciones y opiniones que tienen los estudiantes de bachillerato, progenitores y docentes sobre las elecciones académicas que se realizan en la educación secundaria, haciendo especial hincapié en el papel de los distintos agentes de socialización en esta elección. En este artículo, analizamos el papel que desempeña la variable género en las elecciones académicas tomando como punto de partida las opiniones que manifiestan los distintos agentes implicados.

\section{MÉTODO}

\subsection{Participantes}

Los resultados que presentamos se corresponden con la segunda fase de un estudio del grupo de investigación ASOCED. En la primera fase de la investigación se 
administró un cuestionario a 2.359 estudiantes de $4 .^{\circ}$ de la ESO y a 1.558 estudiantes que cursaban el primer curso del bachillerato científico-tecnológico. En esta primera fase se obtuvo información sobre los factores que influían en el proceso de decisión académica.

Una vez identificados dichos factores, el equipo de investigación abordó la realización de una segunda fase de la investigación para conocer la percepción que tenían los distintos implicados sobre la toma de decisión vocacional. En esta segunda fase han participado 51 estudiantes ( 23 chicas y 28 chicos), 14 padres/ madres (12 madres y 2 padres) y 38 docentes (23 mujeres y 15 varones). Los estudiantes estaban cursando primero de bachillerato en la modalidad científicotecnológica y los docentes impartían docencia en esta modalidad académica.

\subsection{Instrumento de recogida de datos}

La recogida de datos se realizó mediante la técnica del grupo de discusión. Esta aproximación metodológica tiene el potencial de producir modelos conceptuales que maximizan las experiencias de los participantes, dotando a los resultados de una forma de validez interna que es uno de los puntos fuertes de los enfoques cualitativos de investigación. Se organizaron cinco grupos de discusión con estudiantes de diversas zonas geográficas del Principado de Asturias, se realizaron seis grupos de discusión con docentes en los mismos centros en los que se realizaron los grupos focales con estudiantes, añadiéndose un grupo más en la zona central de Asturias. Por último, se realizaron dos grupos con padres/madres, uno en Oviedo y otro en la cuenca minera. La media de asistentes a los grupos de discusión estuvo entre las seis y las ocho personas.

El protocolo de preguntas que se usó ha sido desarrollado por el grupo de investigación. Todos los miembros del equipo, siete personas en total, revisaron el protocolo que luego fue editado y vuelto a revisar en su última versión por los dos miembros del equipo más implicados en la realización de las entrevistas grupales. Se usaron diferentes protocolos con los distintos colectivos. En el caso de los estudiantes se debatieron los siguientes temas: elección de asignaturas y rendimiento académico, asignaturas y creencias de autoeficacia, interés por distintas asignaturas, percepción de los apoyos y las barreras que encuentran en su elección académica, elección de estudios y estereotipos de género. Respecto a los docentes, los temas para el debate fueron: creencias acerca de la enseñanza y el género; ciencia, tecnología y género. Finalmente, los grupos de discusión con los progenitores ahondaron en los siguientes aspectos: elección académica de sus hijos e hijas; percepción del éxito académico de sus hijos e hijas; utilidad de las distintas asignaturas para su futuro profesional; apoyo mostrado a sus hijos e hijas en las decisiones académicas; asignaturas y estereotipos de género. Los guiones de preguntas facilitaron la organización de la información y el análisis de contenido realizado con posterioridad. 
M. C. RODRÍGUEZ MÉNDEZ, J. V. PEÑA CALVO Y O. GARCÍA PÉREZ

\subsection{Procedimiento}

A partir de los contactos previos que se tuvieron con setenta y dos centros de educación secundaria del Principado de Asturias para realizar la primera fase de la investigación, acudimos a aquellos que tuvieron interés por participar en la segunda fase. Las reuniones comenzaron con una exposición de nuestros objetivos. Presentábamos nuestra investigación como un estudio sobre los procesos de toma de decisión que seguían los estudiantes de secundaria cuando debían afrontar elecciones académicas. Este fue el hilo argumentativo usado, de modo que la idea fue plantear la investigación de la manera más amplia posible, evitando la preparación de discursos alejados de la realidad. Además, intentamos transmitir una valoración positiva de sus aportaciones y aclaramos que lo que se dijera, además de ser confidencial, nos interesaba por ser un reflejo de un proceso que se podía generalizar a otras personas. Los grupos de discusión fueron conducidos por dos miembros del equipo y tuvieron una duración aproximada de entre una hora y hora y media.

Las sesiones fueron grabadas y, con posterioridad, fueron transcritas en papel, al tiempo que se realizaban anotaciones de las interrupciones, risas y otras emociones, tono de voz y énfasis dado a determinadas expresiones. La transcripción del audio a texto escrito fue realizada por una empresa especializada, pero uno de los entrevistadores revisó todas las entrevistas para garantizar la fidelidad de la transcripción.

\subsection{Análisis de datos}

Se establecieron tres niveles de análisis de los datos:

- Clasificación temática de las distintas ideas verbalizadas en los grupos de discusión, elaborando un sistema de categorías. Si bien el sistema de categorías se elaboró a posteriori, el guion de preguntas que orientó las discusiones grupales fue el punto de partida para la definición de los conceptos y las categorías. En varias ocasiones se leyó toda la información, y esta tarea se repitió en diversos momentos del proceso de análisis de contenido. También se descartó parte del material recogido por su escasa relevancia. Se solicitó a dos compañeras que estudiaran el sistema de categorías construido y su aplicación a una serie de textos discursivos seleccionados al azar para valorar su aplicabilidad y coherencia. En la Figura 1 se plasma el sistema de categorías elaborado, estableciendo las relaciones entre ellas.

- Descripción del contenido de las transcripciones: inclusión de comentarios de los participantes en relación con la categoría trabajada en cada momento.

- Interpretación teórica del contenido descrito en el segundo nivel de análisis. Se elaboraron conclusiones en relación a los objetivos de la investigación y tomando como punto de referencia los resultados de investigaciones similares. 


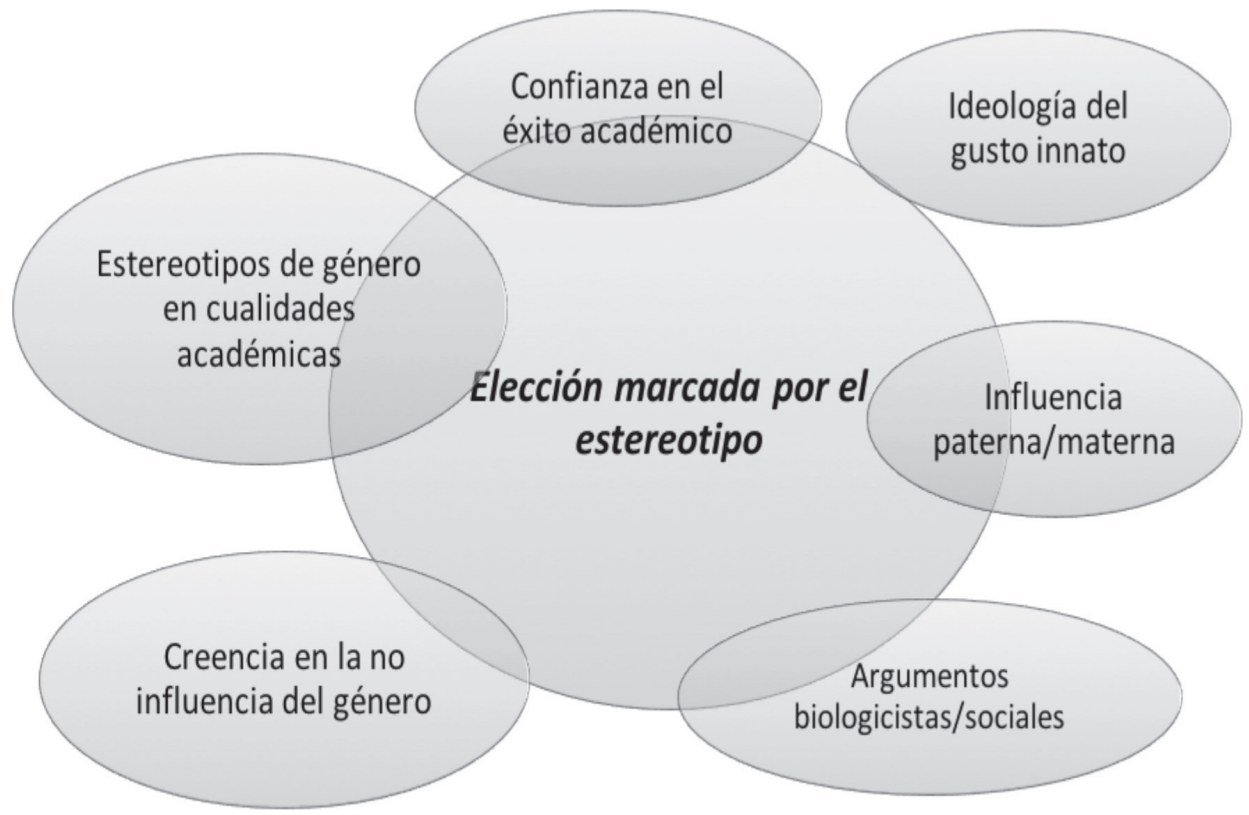

\section{Resultados}

El primer aspecto a destacar es que se observa una claro sesgo de género en la elección. De este modo de las 23 chicas que han participado en los grupos de discusión, 19 se decantan por la modalidad biosanitaria y 4 por la tecnológica. Respecto a los estudiantes varones, 10 de ellos eligen la modalidad biosanitaria y 18 la tecnológica.

Por otro lado, la razón fundamental que indican los estudiantes para elegir la modalidad científico-tecnológica tiene que ver con lo que hemos denominado la «ideología del gusto innato». Según su opinión han elegido estos estudios porque les gustan, siendo muy frecuente que usen expresiones que enfatizan una relación de afinidad con determinadas materias: «siempre me gustó», "nunca me gustó». Del mismo modo, esta ideología del gusto innato también sirve para reforzar determinados estereotipos de género. Además, de las cuatro chicas que se decantan por la modalidad tecnológica, tres de ellas justifican su elección no porque les gusten las asignaturas de la rama tecnológica, sino porque no les gusta la rama biosanitaria, por descarte. Asimismo, la última de ellas indica que ha elegido esa modalidad porque lo relaciona con la posibilidad de optar por salidas profesionales que desarrollan la creatividad y el diseño. Hemos de hacer notar que el padre de esta estudiante es arquitecto. Asimismo, es muy habitual que las chicas justifiquen su 
preferencia por la rama biosanitaria apoyándose en argumentos relacionados con su interés por estas ramas y su escasa o nula preferencia por el campo tecnológico. De esta forma, ambos campos se manifiestan como opuestos e irreconciliables. De forma similar, los progenitores también opinan que sus hijos e hijas poseen una cierta predisposición desde su infancia por las actividades vinculadas a determinadas profesiones, observándose nuevamente un sesgo de género:

Yo en $2{ }^{\circ}$ de la ESO que empecé tecnológica tenía clarísimo que por ahí no. No me gustaba, además sufría (Chica, GD1) ${ }^{1}$.

Desde pequeña ya tenía la mentalidad de que me iba a dedicar a algo relacionado con el itinerario biosanitario porque desde pequeña ya jugaba incluso a ser doctora (Chica, GD5).

La tendencia natural de mi hijo es a las matemáticas, los aparatos, los cacharros, la ciencia, cualquier cosa de ese tema le llama la atención (Madre, GD1).

A mí todo el día me desaparecen destornilladores, no sé dónde están, y aparecen en la habitación de mi hijo; y él de un motor de un coche haz un no sé qué, no sé cómo lo hace... entonces lo veo capacitado (Padre, GD1).

Mi hijo eligió el tecnológico porque yo creo que nació ya siendo así, porque siempre dijo de pequeño que quería trabajar con las manos (Madre, GD2).

En otras ocasiones, y normalmente vinculado a la ideología del gusto innato, algunos estudiantes comentan que su elección también ha estado condicionada por las salidas profesionales de la modalidad. Chicos y chicas usan los mismos argumentos para justificar una elección que está condicionada por el género:

Tampoco sé muy bien lo que hacer pero elegí la rama sanitaria porque creo que tiene más salidas (Chica, GD3).

Escogí el tecnológico porque sentía que tenía más facilidad para este tipo de asignaturas y porque me interesan más las salidas (Chico, GD5).

En línea similar, los estudiantes tienen bastante confianza en terminar los estudios de bachillerato y las carreras universitarias que tienen previsto escoger. De las 20 chicas que contestaron a esta pregunta 12 de ellas manifestaron tener confianza en terminar sus estudios, 6 de ellas no manifestaron ninguna confianza y 2 de ellas se mostraron indecisas. Respecto a los chicos, 22 de ellos respondieron a la pregunta y fueron abrumadora mayoría (20) los que manifestaron su confianza, frente a uno de ellos que tenía confianza en finalizar sus estudios de bachillerato pero no universitarios y uno que se mostró indeciso. Podemos observar cierto desequilibrio por razón de género, pues aunque ambos grupos manifiestan más confianza que la ausencia de ella, los varones tienden, de forma claramente mayoritaria, a ser más confiados en sus posibilidades. Esto se demostró claramente en

1. Cuando se inserten citas textuales de los discursos se incluirá, entre paréntesis, una anotación que contiene el género de la persona y el grupo de discusión en el que participó. 
el caso de un grupo de discusión al que asistían dos gemelos (chico y chica). Ella tenía mejor expediente académico, confirmado verbalmente por el resto de compañeros/as en el grupo de discusión y por el profesor-tutor de ambos estudiantes. Ella comentó que «tenía pensado ser médica pero como vi que era demasiada nota, me planteé que me gustaba enfermería. La carrera de medicina es muy complicada y muy larga». Ella se autoelimina de la opción de medicina porque cree que no va a conseguir la nota necesaria para su acceso. Su hermano, no obstante, está empeñado en hacer medicina cuando es consciente de que "tendré un 6 con algo, casi 7», nota muy alejada de la que deben obtener para acceder a los estudios de medicina. Esta percepción respecto a la mayor inseguridad de las chicas es corroborada por muchos docentes. Estos manifiestan que ellas son más conscientes de sus posibilidades de éxito académico, eligen las opciones académicas teniendo más claras sus aptitudes y capacidades y, quizás, se autoeliminan de determinadas opciones por su inseguridad:

Ellos están más seguros de lo que quieren, más seguros en su mundo.... ellas suelen aspirar a menos. A lo mejor son más conscientes de la realidad (Profesora, GD1).

Ellas a esta edad son más maduras, a lo mejor esto les hace ser más realistas y más conscientes de sus posibilidades (Profesor, GD1).

Son más maduras, las mujeres maduran antes y son más conscientes de lo que se les avecina, de los problemas que van a tener y se les nota bastante (Profesor, GD5).

Quizás la falta de responsabilidad que tienen los chavales es lo que les haga muchas veces... dice: "Yo voy a tirar p'alante». Y sin embargo las chavalas con esa madurez cogen miedo de ir por una ingeniería y se les ponen los pelos de punta (Profesor, GD3).

Asimismo, cuando se pregunta a los estudiantes si el género puede ser un factor que influye en las elecciones académicas presentándoles datos estadísticos que avalan esa elección diferencial, podemos mencionar que, de forma generalizada, contestan que no. Cuando en los grupos se abordaron estas cuestiones, chicos y chicas permanecieron callados, y fue muy difícil estimular al grupo para que manifestara su opinión. La fuerza del estereotipo es tan potente que nunca se han planteado por qué las cosas son como son, simplemente se asumen como "algo normal» en el contexto en el que viven. Tampoco muestran interés por buscar una explicación a este hecho, se asume sin más y opinan que a ellos y ellas no les afecta porque han elegido libremente, sin ningún tipo de condicionamiento, basándose en sus preferencias y gustos individuales. Los argumentos aducidos para afirmar sus posiciones están escasamente fundamentados, pues nunca se lo habían planteado y tampoco habían reflexionado sobre ello. Las cuestiones de género no forman parte de sus preocupaciones y quizás consideren que se trata de un debate superado. En este sentido, el siguiente fragmento discursivo es típico del que se produjo en todos los grupos de discusión. 
M. C. RODRÍGUEZ MÉNDEZ, J. V. PEÑA CALVO Y O. GARCÍA PÉREZ

ESTUDIO CUALITATIVO DE LAS DIFERENCIAS DE GÉNERO EN LA ELECCIÓN DE OPCIONES...

Entrevistadora.- ¿Entonces creéis que el hecho de ser chico o chica acaso no ha influido en vuestra decisión académica o sí lo ha hecho?

Todos.- No.

Entrevistadora.- Pero ¿̇respondéis al patrón?

Todos.- Sí.

Entrevistadora.- Nunca habíais dicho: ¿«Por qué tantos compañeros hacen tecnología y tan pocas chicas no lo hacen»?

Chico.- No.

Chica.-No porque aquí es una cosa normal. Sí, porque siempre fue así, los chicos por tecnología y nosotras por otro lado.

En los casos excepcionales en que se intentó proporcionar una explicación a estas diferencias de género los argumentos oscilaron entre las explicaciones biologicistas basadas en las distintas capacidades que tenemos los unos y las otras para afrontar la realización de determinadas actividades, o argumentos de tipo social basados en la mayor predisposición femenina para elegir ocupaciones vinculadas a tareas de ayuda y cuidado:

Yo creo que tenemos diferentes formas de pensar porque igual sí que es algo, en el cerebro tenemos distintas zonas, la percepción la tienen mejor ellos (Alumna, GD1).

La tecnología siempre se les da mejor a los hombres... Las mujeres éramos nulas (Alumna, GD1)

Creo que es porque a las mujeres se nos da más el ámbito sanitario de tratar con gente, somos más de relaciones (Alumna, GD3).

Porque desde pequeñas las nenas jugamos más como si fueras maestra, enfermera y los nenos más con herramientas, camiones (Alumna, GD1).

En línea con lo que se acaba de mencionar los docentes son poco propensos a recurrir a argumentos de corte biologicista. Sí es cierto que algún docente es de la opinión de que hay diferencias en la capacidad espacial de ambos géneros, teniendo los chicos una mayor habilidad en este sentido. No obstante, esta idea fue marginal y no es representativa de lo que opinan la mayoría de ellos, los cuales se decantan por explicaciones de carácter social. Así, muchos consideran que chicos y chicas se hallan imbuidos por el imaginario social que determina una preferencia de las mujeres y de los varones para distintas profesiones. Afirman que existe una "tradición social» que provoca la emergencia de mensajes distintos para chicos y chicas respecto a lo que es o no apropiado para su género de pertenencia y que estos mensajes tienen un gran poder para condicionar sus decisiones académicas y profesionales:

Ellos asocian la tecnología, la ingeniería con un rol masculino, y hasta que eso no se consiga vencer no habrá una cierta igualdad en el número de alumnos en una asignatura frente a otras (Profesor, GD1). 
Quizás a lo mejor influye la percepción social, la que tienen las chicas del trabajo que van a desarrollar. A lo mejor una chica no se ve en el mundo de una empresa con casco y una llave inglesa... Creo que es la educación social que perciben que les lleva a bascular a las chicas hacia opciones que no son tecnológicas (Profesor, GD2).

Creo que son los roles que están en la sociedad desde la infancia. A las crías se les da la muñeca y a los críos el cochecito, entonces eso condiciona luego las elecciones (Profesor, GD2).

Hay profesiones que son mayoritariamente femeninas y mayoritariamente masculinas y a uno le cuesta verse en una profesión donde eres el único chico o donde ella es la única chica (Profesor, GD4).

Asimismo, la dinámica discursiva que se generó en algunos grupos de discusión respecto a estas cuestiones derivó en una idea que está bastante generalizada entre el alumnado, a saber: la mayor constancia y espíritu de trabajo de las chicas respecto a los chicos. Esta idea también ha sido indicada constantemente en los grupos de discusión con docentes. De este modo, ningún profesor o profesora indica que las chicas tengan menos capacidad para afrontar con éxito las asignaturas del bachillerato científico-tecnológico. Se afirma, con rotundidad, que la capacidad o el rendimiento no depende del género, pero sí perciben, de forma prácticamente unánime, que las chicas son más organizadas, se esfuerzan más en la realización de los trabajos y se toman más seriamente la labor escolar. Por el contrario, los chicos son adjetivados como más desordenados, desmotivados y menos dispuestos a dar prioridad al trabajo escolar:

Estudiamos mejor nosotras a base de poner los codos (Alumna, GD1)

Las chicas maduran antes y son más ordenadas. Nosotros somos más cabeza loca. Sí estudiamos, somos responsables y estudiamos, pero que veo más metidas en materia a las chicas (Alumno, GD3).

Creo que a las chicas se les da mejor estudiar letras porque para estudiar letras no puedes ser vaga y vagos son los chicos, generalmente (Alumna, GD4).

En el tema de estudiar, de empollar letras ellas se esfuerzan más y puede que lo lleven más al día. Nosotros lo vamos dejando, somos más: «Ya lo haremos» (Alumno, GD4).

Las chicas son mucho más cuidadosas. Parece que como tienen esa estrategia de ir haciendo el trabajo día a día y parece que los hombres son más de que solo les importa la nota del examen (Profesora, GD1).

Es que lo notas al llegar al aula como las chicas nada más que te ven cogen inmediatamente se sientan, sacan sus cosas, y los chicos están por allí dándose empujones con ese tipo de actitudes infantiles (Profesora, GD3).

Si analizamos los fragmentos discursivos también observamos la asociación implícita que se hace entre las asignaturas de letras y las mujeres. Se considera que las chicas son mejores en estas materias porque para aprobarlas es necesario ser 
constantes y trabajadoras, cualidades que se asocian al género femenino. En este sentido, es frecuente que los varones consideren que las asignaturas que mejor dominan son las del ámbito científico. Por otro lado, las chicas, pese a haberse decantado por el bachillerato científico-tecnológico, son más variadas en su interpretación. Algunas creen que se les dan mejor las asignaturas de ciencias y otras que las de letras:

Me siento bastante más seguro con las asignaturas de números que con las de letras, porque cuando hay que estudiar teoría me cuesta más (Alumno, GD4).

Yo creo que me siento muy segura en historia porque aunque sea de las teóricas creo que cultura general tengo bastante y también me interesa mucho el temario, sí me siento más segura (Alumna, GD5).

Me siento más segura en las asignaturas teóricas porque si estudiaste sabes que te lo sabes, pero en matemáticas te puedes atascar con un problema. Y más insegura, aunque me guste, puede que en dibujo porque si te dan una pieza que no ves no puedes hacer nada (Alumna, GD5).

Yo me siento más seguro en las asignaturas como matemáticas o dibujo porque son asignaturas que miro el día antes, y, por ejemplo, dibujo basta con entender dos o tres procedimientos y puedes sacar cualquier tipo de ejercicio (Alumno, GD5).

En otro orden de cosas, son muy pocos los estudiantes que relatan situaciones de desacuerdo entre ellos y sus progenitores respecto al proceso de elección vocacional. Los estudiantes manifiestan que su elección es personal, pero que sus padres/madres les apoyan en las decisiones que toman. En aquellos pocos casos en que se han observado desacuerdos estos tienen, casi siempre, un claro sesgo de género. Así las discrepancias se producen con estudiantes que eligen una opción no mayoritaria para su género de pertenencia. No obstante, también debemos destacar el caso de una alumna que se ha decantado por la opción de Medicina, pese a que su padre siempre quiso que hiciese Arquitectura:

Mi madre no me apoya en la elección porque tiré por ciencias. Ella hubiera preferido que escogiera letras porque era más fácil (Alumna, GD1).

Tengo desacuerdos con ellos, sobre todo en la carrera que a mi padre no le gusta mucho y a mi madre sí (Alumno, GD2). Estudiará psicología.

Mi madre siempre me decía que fuera por letras, pero tuve que meterme en el biosanitario (Alumna, GD2).

Los dos esperaban que hiciera una carrera. Mi padre no se habría conformado hasta convencerme de que hiciera una ingeniería (Alumno, GD5).

Mi hijo mucho tiempo quiso ser biólogo marino, no sé muy bien por qué, gracias a Dios, eso se le quitó. Yo quiero que haga una ingeniería (Madre, GD1).

Asimismo, también observamos algunos casos en que padres y madres han actuado como modelos de rol para sus hijos e hijas. Dichos modelos han actuado como referentes que ayudan a mantener los estereotipos de género y, en menos 
ocasiones, como imágenes que favorecen la ruptura de tales estereotipos. Como ejemplos de estudiantes que mantienen una opción estereotipada debemos comentar el caso de una alumna que ha elegido la rama biosanitaria porque quiere hacer enfermería. En este contexto, reflexiona que si su nota no alcanza para entrar en esta carrera universitaria hará magisterio. Preguntada por la entrevistadora por este cambio de elección que no se corresponde con las salidas académicas de la modalidad de bachillerato elegida, nos comenta que: "Mi tía estudió Magisterio y cuando habla de dar clase pues me gusta también lo que hace y cómo vive». Otros ejemplos de elecciones estereotipadas son los casos de estudiantes varones con familiares, normalmente padres, que son ingenieros: "Mi padre es ingeniero ... Creo que si voy a hacer una ingeniería es porque él me ha llevado hasta aquí» (GD5) y otro chico afirma que: "Mi padre estudió ingeniería y siempre como que me llevaron por ese camino". Otro estudiante varón quiere hacer ingeniería y afirma que: "Supongo que tendrá algo que ver que estudie una ingeniería, también la estudió mi padre». Algunos padres y madres comentan que: "En casa, su padre, al haber estudiado una ingeniería... y entonces él lo tiene como entre ceja y ceja el hacerlo» (Madre, GD1); "Yo hice ingeniería, o sea que debo ser una de las excepciones, que de aquella cuando yo estudié había muy pocas y mi idea era que él hiciese también una ingeniería» (Madre, GD1); "Yo tengo amigos, compañeros de mi marido que son los típicos ingenieros y los hijos están cogiendo la carrera de los padres» (Madre, GD2). De forma similar, una alumna afirma que escogió el bachillerato biosanitario porque: "Yo lo escogí porque lo había escogido mi hermana mayor y ya cuando ella estaba estudiando a mí me llamó bastante la atención».

Como referentes que ayudan en una elección no estereotipada por razón de género tenemos el caso de un estudiante varón del GD2 que quiere realizar biotecnología y cuya madre estudió Biología o el caso de un estudiante varón del GD4 que ha elegido el bachillerato biosanitario y cuya hermana está cursando $5 .^{\circ}$ de Medicina. Asimismo, es paradigmático el caso de una estudiante del GD5 que estudiará Arquitectura y reconoce la influencia ejercida por su padre que es arquitecto, manifestando que: "Mi padre, que es arquitecto, nunca me quiso influir con eso, siempre me dijo que estudiase lo que quisiese, pero mi padre y mi madre sí que a la hora de buscar un futuro después del bachiller me aconsejaron que cogiera una carrera con fundamento, digamos, que estudiara en la Universidad».

\section{CONCLUSIONES}

La primera idea que debemos concluir es que los estudiantes de primero de bachillerato científico-tecnológico siguen eligiendo opciones académicas diferenciadas por razón de género, decantándose mayoritariamente las chicas por la rama sanitaria y los chicos por la tecnológica. Asimismo, esta elección está fundamentada en la ideología del gusto innato. De forma similar a otros estudios (Anderson, 2005; Anderson et al., 2008; Linver et al., 2005; Cortés et al., 2012; Rodríguez et al., 2006; Santana et al., 2009; Santana et al., 2012; Papastergiou, 2008), los estudiantes 
y los progenitores opinan que eligen en función de su preferencia e interés por determinadas opciones académicas. No obstante, dicha elección está marcada por el estereotipo y de las respuestas de los entrevistados parece desprenderse la idea de que hay un mayor interés de los chicos por el trabajo manual con máquinas y de las chicas por profesiones vinculadas a la ayuda a otras personas (Reid et al., 2003; Stewart, 1998).

Asimismo, tanto el alumnado como el profesorado entrevistado es unánime al considerar que ellos tienen más confianza en finalizar con éxito sus estudios, que ellas tienen más seguridad en su rendimiento académico en las asignaturas de letras -pese a cursar el bachillerato científico-tecnológico- y que las chicas son más trabajadoras, maduras, responsables y se toman más seriamente la labor escolar. Estos resultados ponen de manifiesto la construcción social de una serie de dicotomías mujer/varón, letras/ciencias, seguridad/inseguridad, esfuerzo escolar/desapego escolar, madurez/inmadurez que los chicos y chicas van asumiendo como propias del mundo que les rodea y que condicionan los pasos que toman en su devenir académico y profesional. Quizás estas potentes asociaciones implícitas son las que hacen muy difícil que el alumnado sea consciente de la importancia del género como factor relevante en la decisión. No son capaces de reflexionar sobre su influencia y son reticentes a concebir otro tipo de explicaciones alternativas más allá del interés o la vocación personal.

En este sentido, adquiere pleno sentido la denominada paradoja "nosotras podemos, yo no puedo». Los estudiantes indican que la desigualdad de género está superada en la sociedad actual (véase también Bovée et al., 2007; Brandell et al., 2008; Johnson, Stone et al., 2008; Meelissen et al., 2008; Papastergiou, 2008), pero, pese a ello, a las chicas les cuesta identificarse personalmente con estas materias, incluso a aquellas que han elegido la modalidad científico-tecnológica. Podemos observar que el estereotipo permanece, pero es difícil hacerlo emerger en los procesos de reflexión colectiva; no obstante, en algunas ocasiones lo hace enmascarado en argumentos de corte biologicista o social. En este sentido, el profesorado mantiene las dicotomías anteriormente mencionadas, pero es más consciente de la discriminación de género y es capaz de construir argumentos más sólidos que la expliquen en base a la "tradición social» (véase también Sainz et al., 2012; Santana et al., 2012).

Respecto a la influencia de los progenitores en la decisión vocacional, los diversos agentes implicados reconocen que la decisión última la adopta el estudiante, si bien contando con el apoyo familiar (véase también Sainz et al., 2012; Santana et al., 2012). En este sentido, han sido poco numerosos los casos de estudiantes que han elegido una opción con la que sus progenitores no estaban de acuerdo, si bien en estos casos se observó que habían hecho una elección no acorde al estereotipo de género. En nuestra opinión, la mayor influencia parental se ha producido a partir del modelado social (véase también Erwin et al., 1998; López, 1995; Zeldin et al., 2000; Zeldin, et al., 2008). Así se relatan numerosos casos de estudiantes cuyos padres/madres tenían una profesión determinada que pudo 
condicionar la elección. Esta situación ha sido especialmente notable en el caso de hijos de ingenieros cuyo deseo es cursar una ingeniería.

Los resultados del estudio cualitativo que hemos realizado confirman la necesidad de incorporar la perspectiva de género en los programas de orientación vocacional. Desde esta perspectiva, diversos estudios aconsejan realizar actividades con los estudiantes de secundaria que muestren modelos femeninos que han tenido éxito en áreas científicas y tecnológicas (Betz, 1992; Papastergiou, 2008). De este modo, se han articulado iniciativas para que mujeres profesionales en estos campos dialoguen con los adolescentes sobre su experiencia de trabajo en ámbitos «masculinos». Otras propuestas organizan conferencias para chicas con mujeres ingenieras o, con un carácter más globalizador, se constituyen campamentos para chicas en los que se desarrollan diversas actividades para aumentar su interés por estos campos profesionales (Kort, 2001; Perham y Pierce, 2001).

De forma complementaria, los programas de orientación académica deben estar diseñados para que los adolescentes puedan discutir y reflexionar sobre los condicionantes de género que influyen en su elección. Hay diversas iniciativas que examinan y discuten con las chicas las razones de su escasa presencia en estos ámbitos profesionales (Betz, 1992; Whiston y Bouwkamp, 2003). En otras ocasiones, el grupo de debate está formado por chicos y chicas, quienes, conjuntamente, dialogan acerca de la influencia del género en sus decisiones vocacionales (Turner y Lapan, 2005). En este sentido, destacamos el programa de orientación "Rompiendo Esquemas» porque ha sido pionero al introducir la perspectiva de género en la orientación vocacional en nuestro país (García y Sánchez, 2007). Por último, mencionar que algunos programas reconocen la influencia que ejercen los progenitores en la toma de decisión vocacional y apuestan por incluirlos en algunas de las sesiones a implementar en los centros de educación secundaria. Así, Vouillot, Blanchard, Marro y Steinbruckner (2004) han diseñado una serie de sesiones organizadas en torno a grupos de discusión de chicas con sus padres y madres en donde se intenta evidenciar el sistema de creencias de los progenitores y analizar la influencia que han tenido en las elecciones vocacionales realizadas por sus hijas.

Respecto a algunas sugerencias para futuras líneas de investigación, podemos indicar tres recomendaciones. En primer lugar, se debería estudiar el funcionamiento de los servicios de orientación de los centros de educación secundaria para examinar el asesoramiento vocacional que realizan y en qué medida implementan líneas de actuación para facilitar la ruptura de estereotipos de género en la elección académico-profesional. En segundo lugar, es preciso investigar el papel orientador que realiza el profesorado, en su doble vertiente de docente y tutor/tutora, para analizar la posible transmisión de estereotipos de género en la orientación vocacional. Finalmente, una línea de investigación que consideramos fructífera tiene como foco de interés las familias del alumnado de educación secundaria. Así, se debe examinar, con más detenimiento, el papel que juegan los progenitores en la elección vocacional de los estudiantes, haciendo especial hincapié en la transmisión, tanto implícita como explícita, de estereotipos sexistas respecto a los estudios y las profesiones. 
M. C. RODRÍGUEZ MÉNDEZ, J. V. PEÑA CALVO Y O. GARCÍA PÉREZ

ESTUDIO CUALITATIVO DE LAS DIFERENCIAS DE GÉNERO EN LA ELECCIÓN DE OPCIONES...

\section{REFERENCIAS BIBLIOGRÁFICAS}

ANDERSON, N. (2005) Redressing the gender imbalance in ICT professions: toward- state-level strategic approaches. Australian Educational Computing, 20 (2), 3-10.

Anderson, N.; Lankshear, C.; Timms, C. y Courtney, L. (2008) «Because it's boring, irrelevant and I don't like computers": why high school girls avoid professionally-oriented ICT subjects. Computers \& Education, 50, 1304-1318. Doi: 10.1016/j.compedu.2006.12.003.

BETZ, N. E. (1992) Counseling uses of career self-efficacy theory. Career Development Quarterly, 41 (1), 22-26. Doi: 10.1002/j.2161-0045.1992.tb00352.x.

Bovée, C.; Voogt, J. y Meelissen, M. (2007) Computer attitudes of primary and secondary in South Africa. Computers in Human Behavior, 23, 1762-1776. Doi: 10.1016/j. chb.2005.10.004.

BRANDELL, G.; LeDER, G. y NySTRÖM, P. (2007) Gender and mathematics: recent development from a Swedish perspective. ZDM Mathematics Education, 39, 235-250. Doi: 10.1007/ s11858-007-0025-4.

BRANDElL, G. y STABerG, E.-M. (2008) Mathematics: a female, male or gender-neutral domain? A study of attitudes among students at secondary level. Gender and Education, 20 (5), 495-509. Doi: 10.1080/09540250701805771.

Clark, J. (2005) Women and science careers: leaky pipeline or gender filter? Gender and Education, 17 (4), 369-386. DOI: 10.1080/09540250500145072.

Clegg, S. y Trayhurn, D. (1999) Gender and computing: not the same old problem. British Educational Research Journal, 26 (1), 75-89. Doi: 10.1080/014119200109525.

CORTÉs, A. y CONCHADO, A. (2012) Los contextos parentales y académicos y los valores laborales en la toma de decisiones en bachillerato. Estudios sobre Educación, 22, 93-114.

ERWIN, L. y MAUTURRO, P. (1998) Beyond access: considering gender deficits in science education. Gender and Education, 10 (1), 51-69. Doi: 10.1080/09540259821096.

Fox, L. H. y Soller, J. F. (2001) Psychological dimensions of gender differences in mathematics, en JACOBS, J.; BeCKer, J. y GILMER, G. (eds.) Changing the faces of mathematics. Virginia, National Council of Teachers of Mathematics, 9-24.

Frome, P.; Alfeld, C.; Eccles, J. y Barber, B. (2006) Why don't they want a male-dominated job? An investigation of young women who changed their occupational aspirations. Educational Research and Evaluation, 12 (4), 359-372. Doi: 10.1080/13803610600765786.

García, G. y SÁncheZ, I. (2007) Rompiendo esquemas. Programa de orientación académica $y$ profesional. Oviedo, Instituto Asturiano de la Mujer.

Johnson, R. D.; Stone, D. L. y Philipps, T. N. (2008) Relations among ethnicity, gender, beliefs, attitudes, and intention to pursue a career in information technology. Journal of Applied Social Psychology, 38 (4), 999-1022. Doi: 10.1111/j.1559-1816.2008.00336.x.

KORT, E. (2001) Role models and real-life experiences. Influencing girl's career choices in mathematics and science, en Jacobs, J.; Becker, J. y Gilmer, G. (eds.) Changing the faces of mathematics. Virginia, National Council of Teachers of Mathematics, 117-123.

LI, Q. (1999) Teachers' beliefs and gender differences in mathematics: a review. Educational Research, 41 (1), 63-76. Doi: 10.1080/0013188990410106.

Linver, M. R. y Davis-Kean, P. E. (2005) The slippery slope: what predicts math grades in middle and high school? New Directions for Child and Adolescent Development, 110, 49-64. Doi: 10.1002/cd.149.

López, M. (1995) La elección de una carrera típicamente femenina o masculina. Desde una perspectiva psicosocial: la influencia del género. Madrid, Ministerio de Educación y Ciencia. 
MeElissen, M. y Drent, M. (2008) Gender differences in computer attitudes: does the school matter? Computers in Human Behavior, 24, 969-985. Doi: 10.1016/j.chb.2007.03.001.

Ministerio de EdUCACIÓn, Cultura y DePORTE (2016) Estadísticas de la educación en España. Recuperado de http://www.mecd.gob.es/servicios-al-ciudadano-mecd/estadisticas/ educacion.html.

Papastergiou, M. (2008) Are computer science and information technology still masculine fields? High school student's perceptions and career choices. Computers $\&$ Education, 51, 594-608. Doi: 10.1016/j.compedu.2007.06.009.

Perham, B. y Pierce, R. (2001) Calculate the possibilities. A program in mathematics and science for young women, en JACOBS, J.; BECKER, J. y GILMER, G. (eds.) Changing the faces of mathematics. Virginia, National Council of Teachers of Mathematics, 151-154.

ReID, N. y SKRYABINA, E. (2003) Gender and physics. International Journal of Science Education, 25 (4), 509-536. Doi: 10.1080/0950069022000017270.

ReINen, I. J. y Plomp, T. (1997) Information technology and gender equality: a contradiction in terminis. Computers \& Education, 28 (2), 65-78.

RodD, M. y BARTHOlOMEW, H. (2006) Invisible and special: Young women's experiences as undergraduate mathematics students. Gender and Education, 18 (1), 35-50. Doi: 10.1080/09540250500195093.

Rodríguez, C. y Angulo, F. (2006) Problemas y limitaciones del acceso de las jóvenes a las tecnologías de la información y la comunicación, en Rodríguez, C. (comp.) Género y currículo. Aportaciones del género al estudio y práctica del currículo. Madrid, Akal, 131-152.

SÁINZ, M. y LóPEZ-SÁEZ, M. (2010) Gender differences in computer attitudes and the choice of technology-related occupations in a sample of secondary students in Spain. Computers E Education, 54 (2), 578-587. Doi:10.1016/j.compedu.2009.09.007.

SÁINZ, M.; MENESES, J. y LÓPEZ, B. (2014) Gender stereotypes and attitudes towards information and communication technology professionals in a sample of Spanish secondary students. Sex Roles, 1-15. Doi: 10.1007/s11199-014-0424-2.

SÁinz, M.; PÁlmen, R. y García-Cuesta, S. (2012) Parental and secondary school teachers' perceptions of ICT professionals, gender differences and their role in the choice of studies. Sex Roles, 66, 235-249. Doi: 10.1007/s11199-011-0055-9.

Santana, L. y Feliciano, L. (2009) Dificultades en el proceso de toma de decisiones académico-profesionales: el reto de repensar la orientación en Bachillerato. Revista de Educación, 350, 323-350.

Santana, L.; Feliciano, L. y Jiménez, A. (2012) Toma de decisiones y género. Revista de Educación, 359, 357-387.

ScotT, A. B. y MAllinckrodt, B. (2005) Parental emotional support, science self-efficacy, and choice of science major in undergraduate women. The Career Development Quarterly, 53, 263-273. Doi: 10.1002/j.2161-0045.2005.tb00995.x.

Singh, K.; Allen, K. R.; Scheckler, R. y Darlington, L. (2007) Women in computer-related majors: A critical synthesis of research and theory from 1994 to 2005. Review of Educational Research, 77 (4), 500-533. Doi: 10.3102/0034654307309919.

Stewart, M. (1998) Gender issues in physics education. Educational Research, 40 (3), 283293. Doi: $10.1080 / 0013188980400302$.

TURNER, S. L. y LAPAN, R. T. (2005) Evaluation of an intervention to increase non-traditional career interest and career-related self-efficacy among middle-school adolescents. Journal of Vocational Behavior, 66, 516-531.Doi:10.1016/j.jvb.2004.02.005. 
VÁzquez, A. y Manassero, M. A. (2007) En defensa de las actitudes y emociones en la educación científica (II): evidencias empíricas derivadas de la investigación. Revista Eureka sobre Enseñanza y Divulgación de las Ciencias, 4 (3), 417-441.

VÁzquez, A. y Manassero, M. A. (2008a) La elección de asignaturas de ciencia: análisis de los factores determinantes. Revista Española de Pedagogía, 241, 541-558.

VÁzQuez, A. y Manassero, M. A. (2008b) La vocación científica y tecnológica de las chicas en secundaria y la educación diferenciada. Bordón, 60 (3), 149-163.

VÁzquez, A. y Manassero, M. A. (2009) Patrones actitudinales de la vocación científica y tecnológica en chicas y chicos de secundaria. Revista Iberoamericana de Educación, 50 (4), 1-15.

Vouillot, F.; Blanchard, S.; Marro, C. y Steinbrukner, M.-L. (2004) La division sexuée de l'orientation et du travail: une question théorique et une question de pratiques. Psychologie du Travail et des Organisations, 10, 277-291. Doi. 10.1016/j.pto.2004.07.004.

Whiston, S. C. y Bouwkamp, J. C. (2003) Ethical implications of career assessment with women. Journal of Career Assessment, 11 (1), 59-75. Doi: 10.1177/106907202237460.

ZARRET, N. R. y MALANCHUK, O. (2005) Who's computing? Gender and race differences in young adult's decisions to pursue an information technology career. New Directions for Child and Adolescent Development, 110, 65-84. Doi: 10.1002/cd.150.

Zeldin, A. L.; Britner, S. L. y PAjares, F. (2008) A comparative study of the self-efficacy beliefs of successful men and women in mathematics, science and technology careers. Journal of Research in Science Teaching, 45 (9), 1036-1058. Doi: 10.1002/tea.20195.

Zeldin, A. L. y PajARES, F. (2000) Against the odds: self-efficacy beliefs of women in mathematical, scientific and technological careers. American Educational Research Journal, 37 (1), 215-246. Doi: 10.3102/00028312037001215. 